Article

\title{
Plasmon-Emitter Hybrid Nanostructures of Gold Nanorod-Quantum Dots with Regulated Energy Transfer as a Universal Nano-Sensor for One-Step Biomarker Detection
}

\author{
Xuemeng $\mathrm{Li}^{1,+}$, Yingshuting Wang ${ }^{1,+}$, Quanying Fu ${ }^{1}$, Yangyang Wang ${ }^{1}$, Dongxu Ma ${ }^{1}$, \\ Bin Zhou ${ }^{1}$ and Jianhua Zhou ${ }^{1,2, *(D)}$ \\ 1 Key Laboratory of Sensing Technology and Biomedical Instruments of Guangdong Province, \\ School of Biomedical Engineering, Sun Yat-sen University, Guangzhou 510275, China; \\ lixuemeng6758@126.com (X.L.); wangysht@mail2.sysu.edu.cn (Y.W.); fuqy3@mail2.sysu.edu.cn (Q.F.); \\ wang45yangyang@163.com (Y.W.); ma023ma@126.com (D.M.); zhoub56@mail2.sysu.edu.cn (B.Z.) \\ 2 Division of Engineering in Medicine, Department of Medicine, Brigham and Women's Hospital, \\ Harvard Medical School, Cambridge, MA 02139, USA \\ * Correspondence: zhoujh33@mail.sysu.edu.cn; Tel./Fax: +86-20-3938-7890 \\ + The authors contributed equally to this work.
}

Received: 10 February 2020; Accepted: 26 February 2020; Published: 1 March 2020

\begin{abstract}
Recently, biosensing based on weak coupling in plasmon-emitter hybrid nanostructures exhibits the merits of simplicity and high sensitivity, and attracts increasing attention as an emerging nano-sensor. In this study, we propose an innovative plasmon-regulated fluorescence resonance energy transfer (plasmon-regulated FRET) sensing strategy based on a plasmon-emitter hybrid nanostructure of gold nanorod-quantum dots (Au NR-QDs) by partially modifying QDs onto the surfaces of $\mathrm{Au}$ NRs. The Au NR-QDs showed good sensitivity and reversibility against refractive index change. We successfully employed the Au NR-QDs to fabricate nano-sensors for detecting a cancer biomarker of alpha fetoprotein with a limit of detection of $0.30 \mathrm{ng} / \mathrm{mL}$, which displays that the sensitivity of the Au NR-QDs nano-sensor was effectively improved compared with the Au NRs based plasmonic sensing. Additionally, to demonstrate the universality of the plasmon-regulated FRET sensing strategy, another plasmon-emitter hybrid nano-sensor of Au nano-prism-quantum dots (Au NP-QDs) were constructed and applied for detecting a myocardial infarction biomarker of cardiac troponin I. It was first reported that the change of absorption spectra of plasmonic structure in a plasmon-emitter hybrid nanostructure was employed for analytes detection. The plasmon-regulated FRET sensing strategy described herein has potential utility to develop general sensing platforms for chemical and biological analysis.
\end{abstract}

Keywords: plasmonic-emitter hybrid nanostructure; localized surface plasmon resonance; fluorescence resonance energy transfer; gold nanorod; quantum dot; biomarker

\section{Introduction}

Plasmonic nanostructures generate extremely confined and enhanced electromagnetic fields as well as display localized surface plasmon resonance (LSPR) peaks in absorption or scattering spectra under the incident light [1-4]. LSPR peaks of plasmonic nanostructures are sensitive to small changes of the adjacent refractive index (RI) caused by the molecular binding events on the metal/dielectric interfaces [5-7]. Therefore, plasmonic nanostructures have lately attracted considerable attention as a new class of simple, rapid, and label-free nano-sensors, which enable many important applications, 
such as biomarkers detection, environmental pollution analysis, and food quality monitoring [8-13]. To exploit new sensing schemes and improve the sensitivity of plasmonic nano-sensors $[14,15]$, efforts have been devoted to the plasmonic hybrid nanostructures, such as plasmon-exciton hybrid nanostructures [16,17], plasmon-dielectric hybrid nanostructures [18], and plasmon-emitter hybrid nanostructures $[19,20]$. Among them, plasmon-emitter hybrid nanostructures exhibit the advantages of simplicity and high sensitivity, and are widely concerned [21,22].

In a plasmon-emitter hybrid nanostructure, the emitter generates fluorescence under the excitation of light, and the plasmonic nanostructure is introduced to regulate the fluorescence intensity due to the weak coupling between the plasmonic nanostructure and the emitter [23,24]. This coupling degree in a plasmon-emitter hybrid nanostructure could be changed not only by the distance between the plasmon and emitter, but also by the degree of spectral overlap between the absorption spectrum of the plasmonic nanostructure and the emission spectrum of the emitter [25-27]. These changes of the coupling degree that depended on the distance or spectral overlap make it easy to achieve biochemical sensing by associating the molecular specific recognition with the previously mentioned properties of plasmon-emitter hybrid nanostructures. To date, the coupling mechanisms of plasmon-emitter hybrid nanostructures, known as nano-sensors, are employed for sensitive and rapid detection of metal ions [28], small molecules [29-31], proteins [32,33], and pathogens [34,35], which have been mainly classified into two types. One is based on plasmon-enhanced fluorescence (PEF) in which the confined electromagnetic fields of the plasmonic nanostructure enhance the fluorescence intensity of the emitter $[14,36]$. The recognition of analytes is designed to enhance the coupling by changing the plasmon-emitter distance, and the fluorescence intensity is further increased. Those nano-sensors possess the advantages of a simple structure and high sensitivity but are confronted with the limitations of compromised reproducibility and time-consuming operations [25]. Another type of plasmon-emitter hybrid nano-sensor relies on fluorescence resonance energy transfer (FRET) in which a non-radiative energy transfer $\left(E_{t}\right)$ from the emitter to the plasmonic nanoparticle is led by the LSPR of the plasmonic nanoparticle [15,37-39]. The recognition of analytes can alter the plasmon-emitter distance or destroy plasmon-emitter hybrid nanostructures, which causes a variation of fluorescence intensity. Those nano-sensors usually work in solutions with the merits of highly sensitive and the convenience of washing free [21]. However, they still suffer from some shortcomings, such as the analytes-dependent design of the sensing system, the complexity of sensor fabrication, and the lack of universality. Therefore, it is still desired to propose novel sensing strategies that benefit the superiorities of nano-photonics to develop biosensors with the merits of easy fabrication, high sensitivity, and certain universality. Herein, we proposed a new sensing strategy based on FRET, defined as plasmon-regulated FRET strategy, which is demonstrated by a plasmon-emitter hybrid nano-sensor based on gold nanorod-quantum dots (Au NR-QDs). As shown in Scheme 1, the plasmon-emitter hybrid nanostructures of Au NR-QDs were prepared by partially linking QDs onto the surface of Au NRs through covalent bonds. The energy of the excited QDs (i.e., emitter) is partly transferred to Au NRs (i.e., plasmon) through a non-radiative FRET process [29], and the remaining radiative energy supports the Au NR-QDs apta-sensor to emit fluorescence. The recognition of molecules by the aptamer could alter the RI $(\Delta n)$ surrounding the Au NR-QDs apta-sensor, which induces the shift of the LSPR peak of Au NR and results in the increase of spectral overlap between the absorption spectrum of Au NR and the emission spectrum of QDs. Then, the regulation of $E_{t}$ is triggered and further leads to the variation of fluorescence intensity for biosensing. Distinguishing from the previously mentioned plasmon-emitter hybrid nano-sensors based on PEF or FRET, our plasmon-regulated FRET nano-sensors have the constant plasmon-emitter distance, and apply the absorption spectrum of the plasmonic nanostructures to regulate the $E_{t}$ for biosensing. 

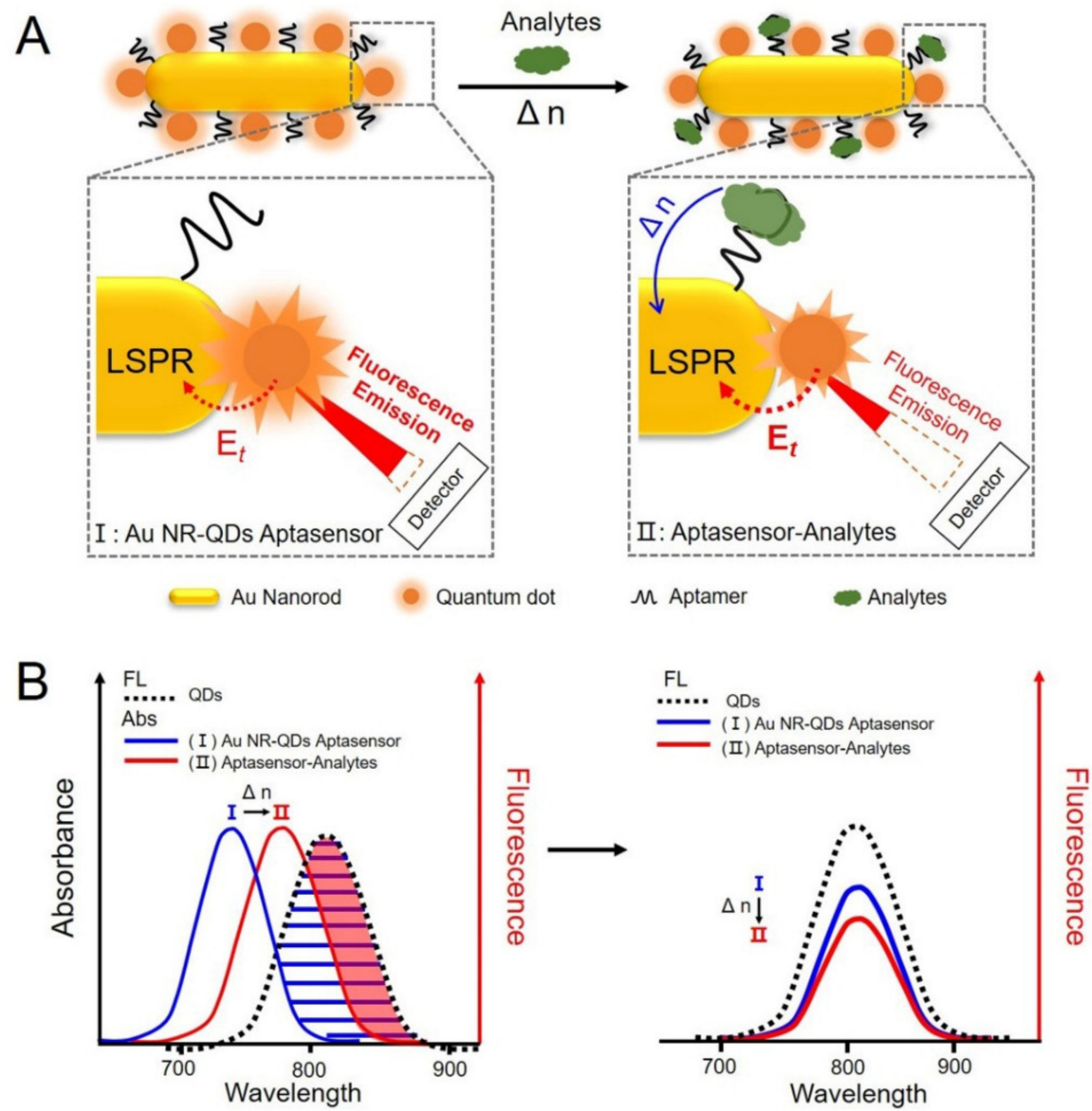

Scheme 1. Schematic illustration of a plasmon-regulated fluorescence resonance energy transfer (plasmon-regulated FRET) sensing system based on the Au nanorod-CdSeTe quantum dots (Au NR-QDs) apta-sensor. (A) The energy transfer $\left(E_{t}\right)$ of the Au NR-QDs apta-sensor before (I) and after (II) capturing analytes. (B) The changes of absorption and fluorescence emission spectra in the same process of (A).

\section{Materials and Methods}

\subsection{Chemicals and Materials}

Chloroauric acid hydrate $\left(\mathrm{HAuCl}_{4} \cdot 3 \mathrm{H}_{2} \mathrm{O}\right.$, Lot\#: F1719017), hexadecyltrimethylammonium bromide (CTAB, Lot\#: E1719115), sodium borohydride $\left(\mathrm{NaBH}_{4}\right)$, L-ascorbic acid (AA), silver nitrate $\left(\mathrm{AgNO}_{3}\right)$, and cysteamine hydrochloride (Lot\#: I1812063) were commercially available from Aladdin (Shanghai, China). Hydrochloric acid $(\mathrm{HCl})$ was provided by the Guangzhou Chemical Reagent (Guangzhou, China). Carboxylic CdSeTe quantum dots (Lot\#: 1977331) were purchased from ThermoFisher Scientific (Shanghai, China). N-(3-dimethylaminopropyl)-N-ethylcarbodiimide hydrochloride (EDC) and N-hydroxysuccinimide (NHS) were purchased from Sigma Aldrich (Shanghai, China). Glutaraldehyde (GA) was acquired from Damao Chemical Reagent (Tianjin, China). The amino-terminated AFP aptamer with the sequence of 5'- $\mathrm{NH}_{2}$-GTGACGCTCCTAACGC TGACTCAGGTGCAGTTCTCGACTCGGTCTTGATGTGGGTCCTGTCCGTCCGAACCAATC-3' from a previous report was synthesized from BGI (Shenzhen, China) [40]. Human alpha fetoprotein (AFP, Lot\#: L2C00201) was supplied by Shanghai Linc-Bio Science Co (Shanghai, China). AFP monoclonal antibody (Lot\#: 2AFP-28) was obtained from Fapon Biotech Inc (Shenzhen, China). Human cardiac troponin I (cTnI, Lot\#: ZKP13) and anti-cTnI monoclonal antibody (Lot\#: XJ235) were all from Tongrenxin Biotechnology (Xiamen, China). 


\subsection{Apparatus}

The UV-vis absorption spectra were recorded by a UV-vis spectrophotometer (L3S, Inesa, Shanghai, China). The fluorescence emission spectra were obtained from a fluorescence spectrophotometer (FS5, Edinburgh Instruments Ltd., Livingston, UK). Transmission electron microscopy (TEM) images were captured by a transmission electron microscope (JEM-1400, JEOL Ltd., Tokyo, Japan) operated at $120 \mathrm{kV}$. Zeta potential measurements were performed on a zeta potential analyzer (ZS90, Malvern Instruments Ltd., Malvern, UK). Deionized water with a resistivity of $18.2 \mathrm{M} \Omega \cdot \mathrm{cm}$ was prepared through a Milli-Q Advantage A10 water system (Millipore, Billerica, MA, USA).

\subsection{Synthesis of $A u N R s$}

The Au NRs stabilized by CTAB were synthesized trough a seed-mediate method with some modification [41]. First, the $10 \mu \mathrm{L}$ of $\mathrm{HAuCl}_{4} \cdot 3 \mathrm{H}_{2} \mathrm{O}(0.125 \mathrm{M})$ was mixed with $4.7 \mathrm{~mL}$ of $0.1 \mathrm{M} \mathrm{CTAB}$ to obtain a homogenous solution under gentle stirring. Then, $0.3 \mathrm{~mL}$ of fresh-prepared ice-cold aqueous $\mathrm{NaBH}_{4}$ solution $(0.01 \mathrm{M})$ was rapidly injected into the mixture. After stirring violently for 2 min, the brownish seed solution was obtained. Fresh seed solution was incubated in a water bath at $30^{\circ} \mathrm{C}$ at about $3 \mathrm{~h}$ and then used for synthesizing Au NRs.

For the synthesis of $\mathrm{Au}$ NRs, growth solution was prepared by adding $0.4 \mathrm{~mL}$ of $0.125 \mathrm{M}$ $\mathrm{HAuCl}_{4} \cdot 3 \mathrm{H}_{2} \mathrm{O}, 0.1 \mathrm{~mL}$ of $1 \mathrm{M} \mathrm{HCl}$, and $0.12 \mathrm{~mL}$ of $\mathrm{AgNO}_{3}$ into $100 \mathrm{~mL}$ of $0.1 \mathrm{M} \mathrm{CTAB}$ solution under continuous gentle stirring. Next, $0.8 \mathrm{~mL}$ of $0.1 \mathrm{M} \mathrm{AA}$ was added, and the color of the mixture changed from orange to colorless. Lastly, $0.24 \mathrm{~mL}$ of seed solution was rapidly injected into the growth solution under vigorous stirring. After stirring for $5 \mathrm{~min}$, the resultant solution was left undisturbed in a $30^{\circ} \mathrm{C}$ water bath overnight to allow the complete growth of Au NRs.

\subsection{Functionalization of Au NRs with Amino Groups}

Amino-modified Au NRs were obtained from literature with some modification [42]. Briefly, as-prepared Au NRs solution was centrifuged at $9000 \times g$ for $30 \mathrm{~min}$ to remove the excess CTAB and ions in the growth solution. The precipitate was then re-dispersed in deionized water. Next, $0.1 \mathrm{~mL}$ of $50 \mathrm{mM}$ cysteamine hydrochloride was added dropwise into $5 \mathrm{~mL}$ of Au NRs solution under sonication at $50{ }^{\circ} \mathrm{C}$ and kept for $0.5 \mathrm{~h}$. After that, the solution was moved to a $50{ }^{\circ} \mathrm{C}$ water bath and kept incubated for $2 \mathrm{~h}$ to allow the complete modification of both sides and tips of the Au NRs. Lastly, as-prepared amino-modified Au NRs solution was centrifuged and washed by deionized water and stored at $4{ }^{\circ} \mathrm{C}$ for further use.

\subsection{Preparation of $A u N R-Q D s$ Assemblies}

A commercial carboxylic CdSeTe quantum dots (QDs) whose emission peak wavelength was $\sim 800 \mathrm{~nm}$ was selected as the fluorescence emitter. Au NR-QDs assemblies were synthesized through an EDC/NHS method [43,44]. A total of $8 \mu \mathrm{M}$ of QDs in borate buffer was diluted to $30 \mathrm{nM}$ by phosphate buffer saline (PBS) buffer (0.01 M, pH 7.4). Then, $100 \mu \mathrm{L}$ of $40 \mathrm{mM}$ of EDC and NHS mixture was added into $5 \mathrm{~mL}$ of QDs solution to effectively activate the carboxylic groups on the surface of the QDs. After gently shaking for $2 \mathrm{~h}, 5 \mathrm{~mL}$ of as-prepared amino-modified Au NRs was added, and the mixture was shaken for $1 \mathrm{~h}$. Lastly, the mixture was left undisturbed overnight under room temperature, and then centrifuged and washed by deionized water for later use.

\subsection{Sensing Behavior of the Plasmon-regulated FRET Sensing System of Au NR-QDs}

The sensing behavior of the plasmon-regulated FRET sensing system of Au NR-QDs was verified in an increasing RI environment. Au NR-QDs solutions were mixed with different glycerol/water mixtures. The RI conditions were set as 1.333, 1.336, 1.337, 1.339, 1.340, 1.341, 1.343, and 1.344, and the corresponding glycerol/water ratios were $0,2.46 \%, 3.65 \%, 4.81 \%, 5.94 \%, 7.04 \%, 8.12 \%$, and $9.18 \%$, 
respectively. Three measurements were operated at each RI condition to determine the standard deviation (S.D.) of peak wavelength and fluorescence intensity.

\subsection{Reversible RI-Dependent Properties of $A u$ NR-QDs}

The measurements of reversible RI-dependent properties of Au NR-QDs were performed as follows. First, Au NR-QDs solution was mixed with a certain amount of glycerol solution to obtain an RI value of 1.334 as the initial solution. The above Au NR-QDs solution was added with more glycerol solution to increase the RI value to 1.339, and then Au NR-QDs/glycerol solution with RI of 1.339 was added to the pure Au NR-QDs mixture to decrease the RI to 1.334. All of the above operations were repeated three times. The corresponding UV-Vis and fluorescence spectra were recorded after each operation.

\subsection{Fabrication of $A u$ NR-QDs Based Nano-sensors}

The surfaces of Au NRs were modified with aptamers through a cross-linking method by GA [45]. First, $50 \mu \mathrm{L}$ of GA solution ( $50 \mathrm{wt} \%$ ) was added to $5 \mathrm{~mL}$ of Au NR-QDs solution and incubated for $30 \mathrm{~min}$ with gentle shaking. Then, the suspension was purified to get rid of the redundant GA, and re-dispersed in $2.5 \mathrm{~mL}$ of deionized water. Subsequently, $2.5 \mu \mathrm{L}$ aptamers solution $(100 \mu \mathrm{M})$ was dropped into the GA-functionalized Au NR-QDs mixture and incubated at room temperature for $2 \mathrm{~h}$ under gentle shaking. Antibodies (Ab) modified Au NR-QDs nano-sensor for cTnI detection was constructed by the following protocol. Furthermore, $2.5 \mu \mathrm{L} \mathrm{cTnI}$ antibody solution $(2.273 \mathrm{mg} / \mathrm{mL})$ was dropped into the $2.5 \mathrm{~mL} \mathrm{GA}$-functionalized Au NR-QDs mixture and incubated at room temperature for $2 \mathrm{~h}$ under gentle shaking.

\subsection{Detection of AFP Using the Au NR-QDs Apta-sensor}

For AFP detection, AFP stock solution was diluted into different concentrations by PBS, and those diluent AFP solutions were added into $600 \mu \mathrm{L}$ of Au NR-QDs apta-sensor solution. The final concentrations of AFP were $0.1,0.3,1,3$, and $10 \mathrm{ng} / \mathrm{mL}$, respectively. After each operation, solutions were incubated at room temperature for $30 \mathrm{~min}$ and the fluorescence spectra were carried out. The limit of detection (LOD) of the apta-sensor was calculated according to the $3 s_{b} / m$ criterion, where $m$ is the slope of the first three experimental data and $s_{b}$ is the standard deviation of the blank $(\mathrm{n}=3)$. The selectivity of the Au NR-QDs apta-sensor against other agents in serum was tested. As-prepared Au NR-QDs was incubated with PBS, bull serum albumin (BSA, $1 \mu \mathrm{g} / \mathrm{mL})$, glucose $(100 \mathrm{ng} / \mathrm{mL}$ ), and $\operatorname{AFP}(10 \mathrm{ng} / \mathrm{mL})$ at room temperature, respectively. After incubation for $30 \mathrm{~min}$, the fluorescence spectra were recorded.

\subsection{Detection of AFP in Spiked Serum Samples}

The AFP spiked serum samples were prepared by diluting AFP negative serum for 10-fold by PBS, and spiking with standard solution of AFP to obtain the final concentration from 0 to $6.4 \mathrm{ng} / \mathrm{mL}$, respectively. All spiked serums were aged at room temperature for $10 \mathrm{~min}$ before use. Lastly, $10 \mu \mathrm{L}$ of each spiked serum sample was incubated with $600 \mu \mathrm{L}$ of Au NR-QDs apta-sensor solution for $30 \mathrm{~min}$, and then the fluorescence spectra were recorded.

\subsection{Detection of cTnI Using the Antibodies Modified Au NR-QDs and Au NP-QDs Nano-sensors}

Antibodies modified Au NP-QDs nano-sensors were constructed by adding cTnI antibody ( $85 \mu \mathrm{L}$, $2.273 \mathrm{mg} / \mathrm{mL}$ ) into $2.5 \mathrm{~mL}$ of GA-functionalized Au NP-QDs mixture. For cTnI detection, cTnI stock solution was diluted into different concentrations by PBS, and those diluent cTnI solutions were added into $600 \mu \mathrm{L}$ of $\mathrm{Au}$ NR-QDs nano-sensor solution (the final concentrations of cTnI were $0.3,1,3,10$, and $15 \mathrm{ng} / \mathrm{mL}$ ) or Au NP-QDs nano-sensor solution (the final concentrations of cTnI were 2, 4, 6, 8, 
and $10 \mathrm{ng} / \mathrm{mL}$ ), respectively. At each operation, the solutions were incubated with sensors at room temperature for $30 \mathrm{~min}$, and the corresponding fluorescence spectra were recorded, respectively.

\section{Results and Discussion}

\subsection{Fabrication and Characterization of the Plasmon-Emitter Hybrid Nanostructures of Au NR-QDs Assemblies}

Figure 1 shows that plasmon-emitter hybrid nanostructures of Au NR-QDs assemblies could be prepared with our synthesized Au NRs and commercial carboxyl-modified CdSeTe quantum dots (QDs). To fabricate Au NR-QDs assemblies, Au NRs with merits of easy preparation and adjustable absorption spectra are chosen as plasmonic nanostructures [46,47]. CdSeTe QDs with the narrow emission peak are selected as emitters [48]. The fabrication process of Au NR-QDs is illustrated in Figure 1A. First, Au NRs were synthesized by a seed-mediated growth method and then modified by cysteamine to obtain $\mathrm{Au} N \mathrm{NR}_{\mathrm{N}} \mathrm{NH}_{2}$, as previously reported [41,42], and the successful synthesis of $\mathrm{Au}$ NRs- $\mathrm{NH}_{2}$ was proven by a blueshift of LSPR peak (Figure S1) and a clear decrease of the zeta-potential from $+35.3 \mathrm{mV}$ to $+21.2 \mathrm{mV}$ after the modification of cysteamine. Then, Au NR-QDs were formed by

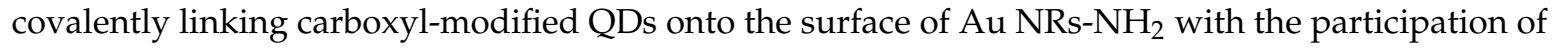
EDC/NHS. The interparticle distance between Au NRs and QDs is regarded as a constant due to the immobilization of covalent bonds.
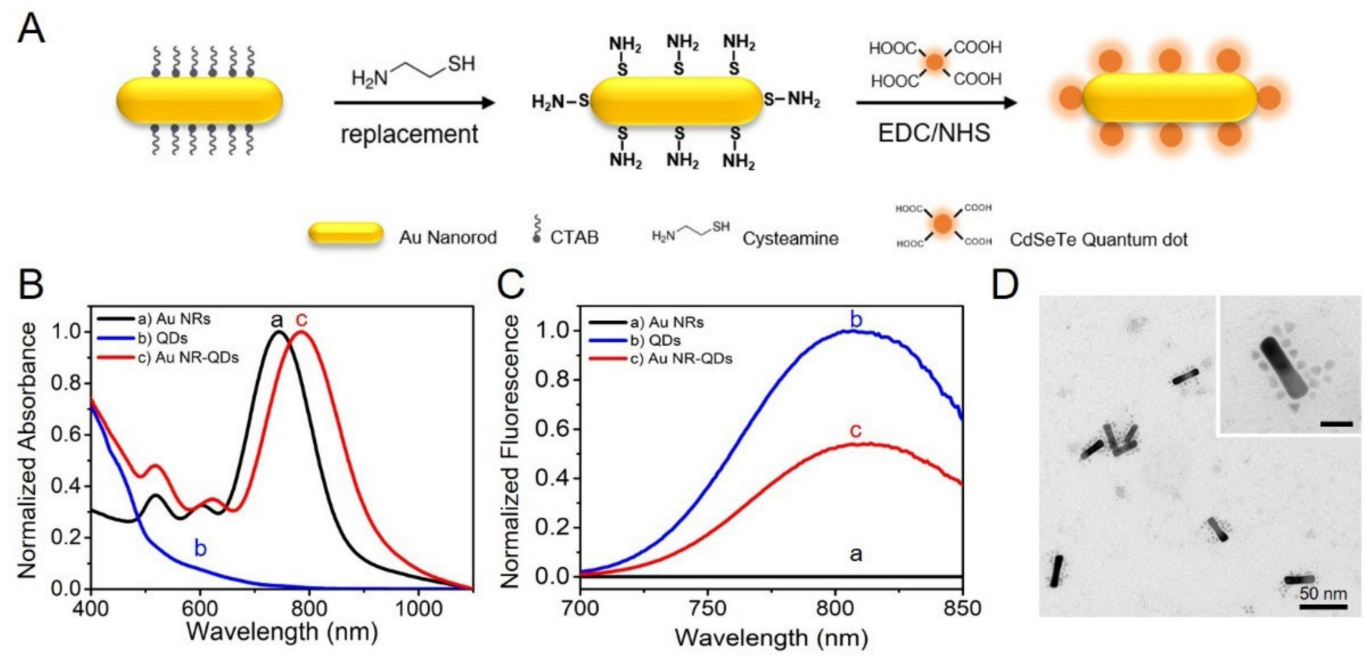

Figure 1. The fabrication and characterization of the plasmon-emitter hybrid nanostructures of Au NR-QDs assemblies. (A) The schematic illustration for the fabrication process of Au NR-QDs.

(B) Normalized UV-vis absorption spectra of the Au NRs (a), QDs (b), and as-prepared Au NR-QDs (c).

(C) Normalized fluorescence spectra of Au NRs (a), QDs (b), and Au NR-QDs (c) in the same process of

(B), where the maximum fluorescence intensity of QDs was regarded as 1.0. (D) Transmission electron microscopy (TEM) images of Au NR-QDs. The inset is a single Au NR-QDs, and the scale bar is $25 \mathrm{~nm}$.

The fabrication of Au NR-QDs was conducted by a series of experiments to ascertain optimal conditions (Figures S2-S5). Au NR-QDs assemblies were fabricated by covalently linking QDs (emission peak of $812 \mathrm{~nm}$ ) with Au NRs $\left(\lambda_{\text {LSPR }}\right.$ of $744 \mathrm{~nm}$ ), and the molar ratio of Au NRs: QDs was 1:130. Normalized absorption spectra of Au NRs (a), QDs (b), and Au NR-QDs (c) are shown in Figure 1B. After Au NRs were modified with QDs, a visible redshift of $\lambda_{\text {LSPR }}$ of Au NRs from $744 \mathrm{~nm}$ to $786 \mathrm{~nm}$ was observed, while the absorption of QDs was negligible between 700 to $800 \mathrm{~nm}$. It seems that the linkage of QDs evidently increased the surrounding RI near the surfaces of Au NRs and caused the redshift of $\lambda_{\text {LSPR }}$ [49]. Figure $1 C$ is the corresponding fluorescence spectra. QDs displayed a fluorescence emission peak at $812 \mathrm{~nm}$ under the excitation light of $450 \mathrm{~nm}$. The fluorescence intensity was quenched dramatically ( $45 \%)$ after linking Au NR with QDs, while Au NRs showed no fluorescent signal. It is reasonable that the fluorescence quenching of Au NR-QDs compared to 
QDs is induced by the occurrence of non-radiative $E_{t}$ from QDs to Au NRs [29,37]. Figure 1D presents the TEM images of Au NR-QDs. The aspect ratio of the Au NR-QDs based on the TEM images is calculated as $\sim 2.1$, with the length of $74 \pm 2 \mathrm{~nm}$ and the diameter of $35 \pm 2 \mathrm{~nm}$. The surfaces of Au NRs were partially and dispersedly coated by $10 \pm 2$ of QDs with uniform distribution, which was direct evidence for the successful fabrication of Au NR-QDs. Moreover, the zeta-potentials declined from $+21.2 \mathrm{mV}$ of Au NRs-NH $\mathrm{N}_{2}$ to $-23.7 \mathrm{mV}$ of Au NR-QDs, which can prove the successful fabrication of $\mathrm{Au}$ NR-QDs (Table S1). Taking the advantage of partial coverage of QDs on the surfaces of Au NRs, the residual surfaces of Au NRs can effectively sense the variation of surrounding RI and be further modified to fabricate RI-sensitive nano-sensors.

\subsection{Sensing Behavior of the Plasmon-Regulated FRET Sensing System of Au NR-QDs}

Figure 2 demonstrates the RI sensitivity for the plasmon-regulated FRET sensing system of $\mathrm{Au}$ NR-QDs. Absorption and fluorescence spectra of Au NR-QDs in different water-glycerol mixtures (the environmental RI increased from 1.333 to 1.344) were recorded accordingly. The $\lambda_{\text {LSPR }}$ of $A u$ NR-QDs apparently red-shifted with the increase of RI (Figure 2A). A linear dependence was observed between the shift of $\lambda_{\mathrm{LSPR}}\left(\Delta \lambda_{\mathrm{LSPR}}\right)$ and environmental RI $\left(R^{2}=0.99\right)$ in Figure 2B. The slope of the fitting line indicated that the RI sensitivity of Au NR-QDs was $\sim 365 \mathrm{~nm} / \mathrm{RIU}$, which was equivalent to that of the bare Au NRs ( $\sim 363 \mathrm{~nm} / \mathrm{RIU})$ in Figure S6. Fluorescence spectra and corresponding maximum fluorescence intensities against varied environmental RI were shown in Figure 2C,D, respectively. The fluorescence intensity of Au NR-QDs showed a decrease with increasing RI, and the change of fluorescence intensity was plotted as a power function of the RI $\left(F / F_{0}=0.624(n-1.332)^{-0.06}, R^{2}=0.95\right)$, which was distinct from the tendency of $\Delta \lambda_{\mathrm{LSPR}}$ in Figure 2B. This power function suggests that our Au NR-QDs system holds higher sensitivity with smaller RI compared to the sensing based on the shift of $\lambda_{\text {LSPR }}$. Figure S7 shows that the fluorescence intensity of QDs was relatively stable in varied RI, which indicates that the decrease of fluorescence intensity is mainly attributed to the effect of Au NRs rather than the fluorescent property of QDs themselves.

In principle, the decrease of fluorescence intensity of Au NR-QDs is due to the variation of $E_{t}$. According to the FRET theory, the level of $E_{t}$ is positively related to the spectral overlap between the absorption of $\mathrm{Au}$ NRs and the emission of QDs. Then the relationship between spectral overlap $\left(S_{O L}\right)$ and relative fluorescence $\left(F / F_{0}\right)$ upon the change of RI $(n)$ was further discussed, where $F_{0}$ is the fluorescence intensity of the Au NR-QDs under an initial RI condition (see Supporting Information).

For a FRET system that holds a large level of $E_{t}$ efficiency, $F / F_{0}$ can be calculated as follows:

$$
\frac{F}{F_{0}}=\frac{n^{4}}{\mathrm{~A} S_{O L}}
$$

where $\mathrm{A}$ is a constant and $\mathrm{A}=\frac{n_{0} 4}{S_{O L O}}$, where $n$ is the RI of the medium and $S_{O L}$ expresses the spectral overlap integral between the normalized absorption of plasmonic structures and emission of emitters. Besides, $n_{0}$ is the surrounding RI of the reference fluorescence intensity $\left(F_{0}\right)$, and $S_{O L 0}$ is the corresponding spectral overlap integral. 

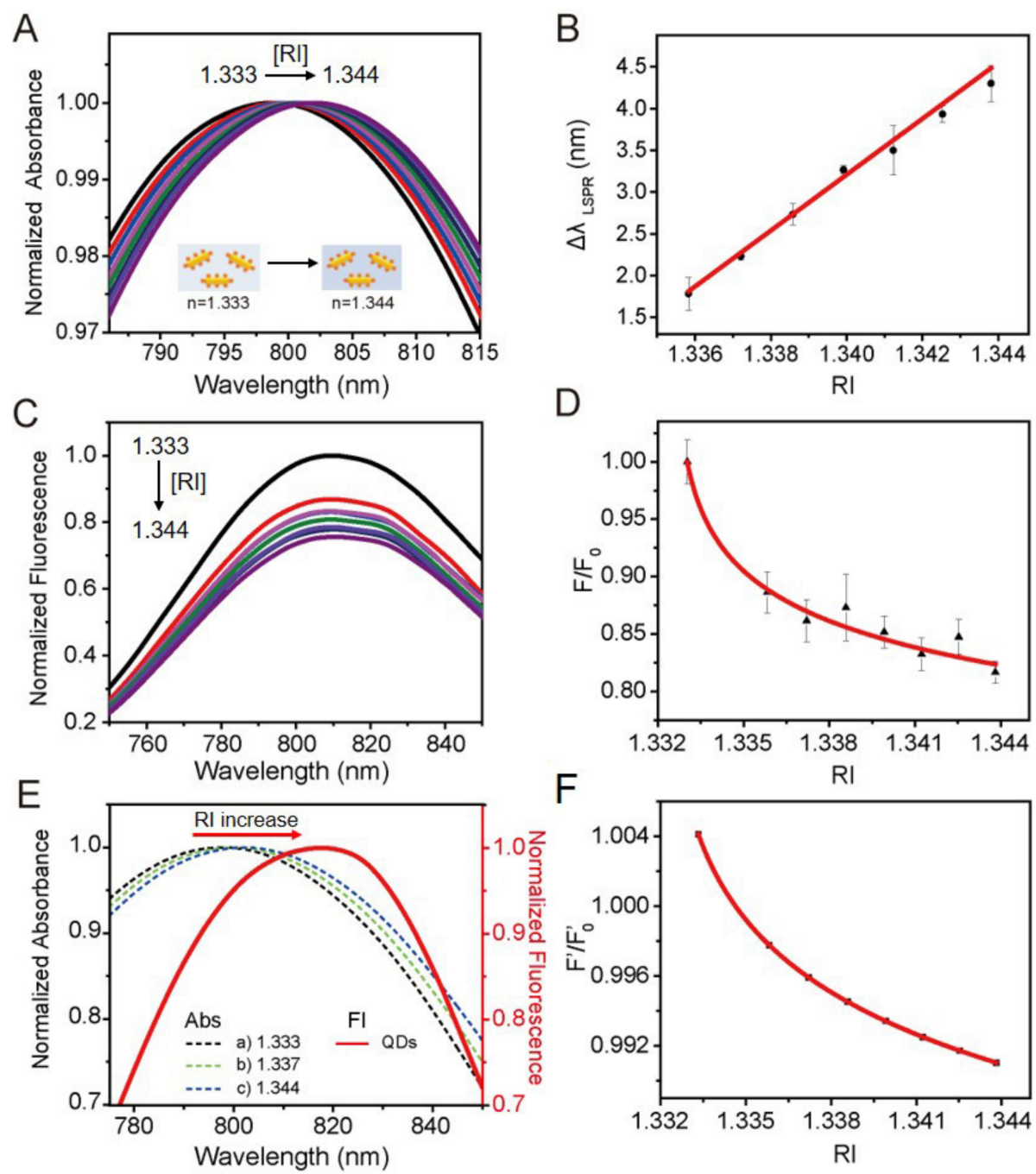

Figure 2. The response to refractive index (RI) for the plasmon-regulated FRET sensing system of Au NR-QDs. (A) Normalized UV-vis absorption spectra of Au NR-QDs in solutions with varying concentrations of water-glycerol mixtures with tuning environmental RI $(n=1.333,1.336,1.337,1.339$, 1.340, 1.341, 1.343 and 1.344), respectively. (B) The $\Delta \lambda_{\text {LSPR }}$ for Au NR-QDs as a linear function of RI $\left(R^{2}=0.99\right)$ extracted from (A). (C) Normalized fluorescence spectra of Au NR-QDs in the same process as (A), where the maximum fluorescence intensity of Au NR-QDs when $n=1.333$ was regarded as 1.0. (D) Relationship between the relative fluorescence intensity $\left(F / F_{0}\right)$ and environmental RI $\left(F / F_{0}=0.624(n-1.332)^{-0.06}, R^{2}=0.95\right)$. (E) Normalized absorption of Au NR-QDs with environmental RI of $1.333,1.337$, and 1.344, respectively, and the normalized fluorescence spectra of QDs. (F) Correlation between theoretical $F / F_{0}\left(F^{\prime} / F_{0}^{\prime}\right)$ and environmental RI.

The normalized absorption spectra of Au NR-QDs in different RI conditions ( $n=1.333,1.337$ and 1.344) and the normalized fluorescence emission spectrum of pure QDs were displayed in Figure 2E. With the RI increasing from 1.333 to 1.344 , a clear increase of $S_{O L}$ can be observed along with the redshift of $\lambda_{\text {LSPR. }}$. A positive correlation between $S_{O L}$ and $n$ was found $\left(S_{O L}=85.83(n-1.332)^{0.006}, R^{2}=0.99\right)$, and $\mathrm{S}_{\mathrm{OL} 0}$ is calculated as 84.481 by the above function when $\mathrm{n}_{0}$ is set up as 1.333 (see Supporting Information). To calculate the theoretical $F / F_{0}$ (i.e., $F^{\prime} / F^{\prime}$ ) in the RI range from 1.333 to 1.344 , the above function $\left(S_{O L}=85.83(n-1.332)^{0.006}\right)$ and the values of $n_{0}$ and $S_{O L 0}$ were substituted into Equation (1). As shown in Figure $2 \mathrm{~F}$, the relationship between $F^{\prime} / F^{\prime}{ }_{0}$ and RI can be approximately equal to $F^{\prime} / F^{\prime}{ }_{0}=0.965(n-1.332)^{-0.006}$ (see Supporting Information), which is consistent with the change tendency of the experimental $F / F_{0}$ in Figure 2D. The results confirm that the change of fluorescence intensity of the plasmon-regulated FRET sensing system is closely related to the variation of the LSPR properties 
of the plasmonic nanostructure, which is sensitive to the surrounding RI change [50]. Additionally, the value of the exponent for the change tendency of $F / F_{0}$ is directly related to the RI sensitivity of LSPR property for the Au NR-QDs. Moreover, the Au NR-QDs exhibited good sensing reversibility and stability upon the change of RI between 1.333 and 1.339 for three cycles (Figure S8). Therefore, this plasmon-regulated FRET sensing system based on Au NR-QDs is capable of having a sensitive and reversible response to the environmental RI, which holds great potential for RI-based sensing of analytes.

\subsection{Construction of the Au NR-QDs Nano-sensor}

To demonstrate the sensing ability of Au NR-QDs for analytes detection, an Au NR-QDs apta-sensor for AFP detection was constructed by modifying Au NR-QDs with aptamers by linking GA (Figure 3A). Aptamers are short and single-strand oligonucleotides that can tightly bind to a broad range of targets (e.g., proteins, drugs, metal ions), and have considerable advantages such as low cost, high affinity, good selectivity, easy labeling, and long-term chemical stability [51-53]. AFP is an important and commonly concerned biomarker for early diagnosis of cancers, especially for hepatocellular carcinoma (HCC) [35]. The concentration of AFP in healthy human plasma is usually less than $25 \mathrm{ng} / \mathrm{mL}$ and increases dramatically when HCC occurs [11]. As shown in Figure 3A, an Au NR-QDs apta-sensor can be constructed by immobilizing amino-terminated aptamers on the surfaces of Au NRs through the linkage of GA. The same modification protocol for various analytes detection can also be applied to other amino-terminated molecules such as antibodies.

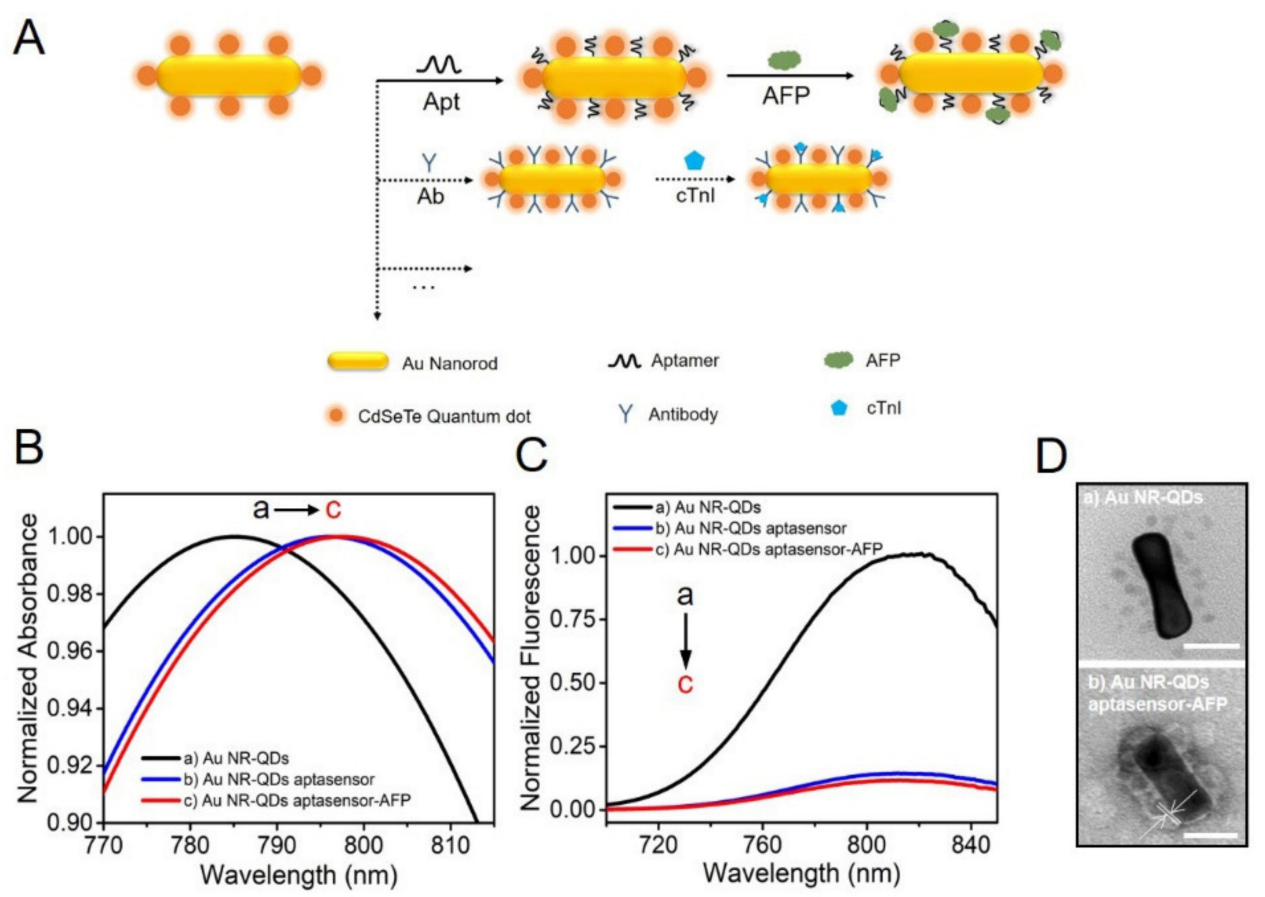

Figure 3. The Au NR-QDs apta-sensor for alpha fetal protein (AFP) detection. (A) Schematics for the construction of Au NR-QDs nano-sensors by modifying Au NR-QDs with different types of sensing layers such as aptamers and antibodies. (B) Normalized UV-vis absorption spectra and (C) normalized fluorescence spectra of Au NR-QDs (a), Au NR-QDs apta-sensor (b), and Au NR-QDs apta-sensor-AFP nanocomplex (c), where the maximum fluorescence intensity of Au NR-QDs was regarded as 1.00. (D) TEM images of Au NR-QDs (a) and Au NR-QDs apta-sensor-AFP nanocomplex (b), respectively. Scale bars are $25 \mathrm{~nm}$. 
The absorption and fluorescence spectra for the construction and application of Au NR-QDs apta-sensor were recorded in Figures $3 \mathrm{~B}$ and $3 \mathrm{C}$, respectively. After the modification of aptamers, a visible redshift of $\lambda_{\mathrm{LSPR}}(10.9 \mathrm{~nm})$ and a dramatic decline of fluorescence intensity $(\sim 85 \%)$ were observed. The zeta-potential declined from $-23.7 \pm 1.4 \mathrm{mV}$ to $-30.2 \pm 3.2 \mathrm{mV}$ after the modification of negative-charged aptamers on the surfaces of Au NR-QDs. All the above results confirmed that an $\mathrm{Au}$ NR-QDs apta-sensor was successfully obtained. Moreover, the negative-charged QDs and aptamers on the surfaces of $\mathrm{Au}$ NR-QDs would keep our Au NR-QDs apta-sensor stable and prevent the sensor from aggregation in the salt solution, which is important for sensing applications [54-57].

Then, the Au NR-QDs apta-sensor was applied to detect AFP of $20 \mathrm{ng} / \mathrm{mL}$. After capturing AFP onto the $\mathrm{Au}$ NR-QDs apta-sensor, another redshift of $\lambda_{\mathrm{LSPR}}$ for $1.2 \mathrm{~nm}$ was measured in the absorption spectra, and the corresponding fluorescence spectra also showed a visible decrease ( 18\%). Compared to the linkage of the aptamer, smaller $\Delta \lambda_{\text {LSPR }}$ and a lower decline of fluorescence intensity occurred after capturing AFP. The reason is that, according to the evanescent field theory, the enhanced electric fields of Au NRs driving from LSPR decay rapidly with distance from the Au NRs surfaces $[58,59]$. The distance between the AFP protein and the Au NR-QDs apta-sensor is farther than the distance between the aptamer and Au NR-QDs, which lead to a lower perturbation of the electric field and are shown as a smaller spectral response. The TEM images of Au NR-QDs (a) and Au NR-QDs apta-sensor-AFP (b) were shown in Figure 3D, respectively. After negative-stained preparations, a transparent white layer $(1.9 \pm 0.3 \mathrm{~nm})$ was observed on the surface of Au NR-QDs (b), which was assumed to be the AFP protein that was captured. The stability of the Au NR-QDs apta-sensor was also studied, and the fluorescence intensity of the Au NR-QDs apta-sensor was kept stable in $3 \mathrm{~h}$ (Figure S9). We successfully obtained an Au NR-QDs apta-sensor and it can be further applied for one-step detection of AFP.

\subsection{Au NR-QDs Apta-sensor for AFP Detection}

The sensitivity of as-prepared Au NR-QDs apta-sensor for AFP detection was further investigated. $\mathrm{Au}$ NR-QDs apta-sensors were incubated with AFP from 0 to $10 \mathrm{ng} / \mathrm{mL}$, and the corresponding fluorescence intensity clearly declined with the ascend of AFP concentration (Figure 4A). A power function dependence was also observed between $F / F_{0}$ and the concentration of AFP $\left(F / F_{0}=0.920\left(C_{A F P}\right.\right.$ $+0.040)^{-0.023}, R^{2}=0.93$ ) in Figure $4 \mathrm{~B}$, which followed the similar change tendency as the RI response experiment in Figure 2. The $10 \mathrm{ng} / \mathrm{mL}$ of AFP was selected as the upper concentration when under incubation with $\mathrm{Au}$ NR-QDs apta-sensors because the changes of fluorescence intensity at AFP concentrations that was higher than $10 \mathrm{ng} / \mathrm{mL}$ were almost the same to the concentration of $10 \mathrm{ng} / \mathrm{mL}$ (unshown data). This indicates that the ability of Au NR-QDs apta-sensors to capture AFP may be saturated around the AFP concentration of $10 \mathrm{ng} / \mathrm{mL}$. The limit of detection (LOD) of AFP was calculated as $0.30 \mathrm{ng} / \mathrm{mL}$, which was far below the critical AFP concentration for early diagnoses of HCC (i.e., $25 \mathrm{ng} / \mathrm{mL}$ [11]) while an Au NRs apta-sensor was also constructed for AFP detection and showed almost no response to AFP from 0 to $50 \mathrm{ng} / \mathrm{mL}$, which suggests that the sensing system of $\mathrm{Au}$ NRs based on $\Delta \lambda_{\text {LSPR }}$ is not sensitive enough to detect AFP for early HCC diagnoses (Figure S10). Thus, our plasmon-regulated FRET sensing strategy can effectively improve the sensitivity of the plasmonic biosensor and realize the one-step detection of AFP.

Furthermore, the selectivity of the Au NR-QDs apta-sensor for AFP detection is also evaluated by incubating Au NR-QDs apta-sensors with PBS, bull serum albumin (BSA), glucose, and AFP under the same condition, respectively. As shown in Figure 4C, the capture of AFP could cause a remarkable decrease of fluorescence intensity $(p<0.001)$, while the variations of fluorescence intensity were negligible $(p>0.05)$ in groups of these interfering agents with higher concentrations. The results confirm the sufficient sensitivity and selectivity of as-prepared Au NR-QDs apta-sensor for AFP detection.

We further applied an as-prepared Au NR-QDs apta-sensor to practical detection of AFP in spiked serum samples. Standard solutions of AFP with different concentrations were spiked into negative serums respectively and then incubated with Au NR-QDs apta-sensor (the final concentration is from 0 
to $6.4 \mathrm{ng} / \mathrm{mL}$, Figure 4D). The fluorescence intensity showed a similar change with the increase of AFP concentration as the detection of AFP in water $\left(F / F_{0}=0.966\left(C_{A F P}+0.064\right)^{-0.020}, R^{2}=0.80\right)$. The LOD for AFP detection in spiked serum sample was calculated to be $1.44 \mathrm{ng} / \mathrm{mL}$, which effectively meets the need for clinical detection of AFP. Additionally, the concentrations of AFP in three spiked serum samples were determined, and the results were summarized in Table 1. Recoveries of known spiked amounts of AFP in three different serum samples were between 104.3\% and 118\% with the relative standard deviation (RSD) being lower than 10\%, which suggests the potential applicability of the Au NR-QDs apta-sensor for clinical detection of AFP.
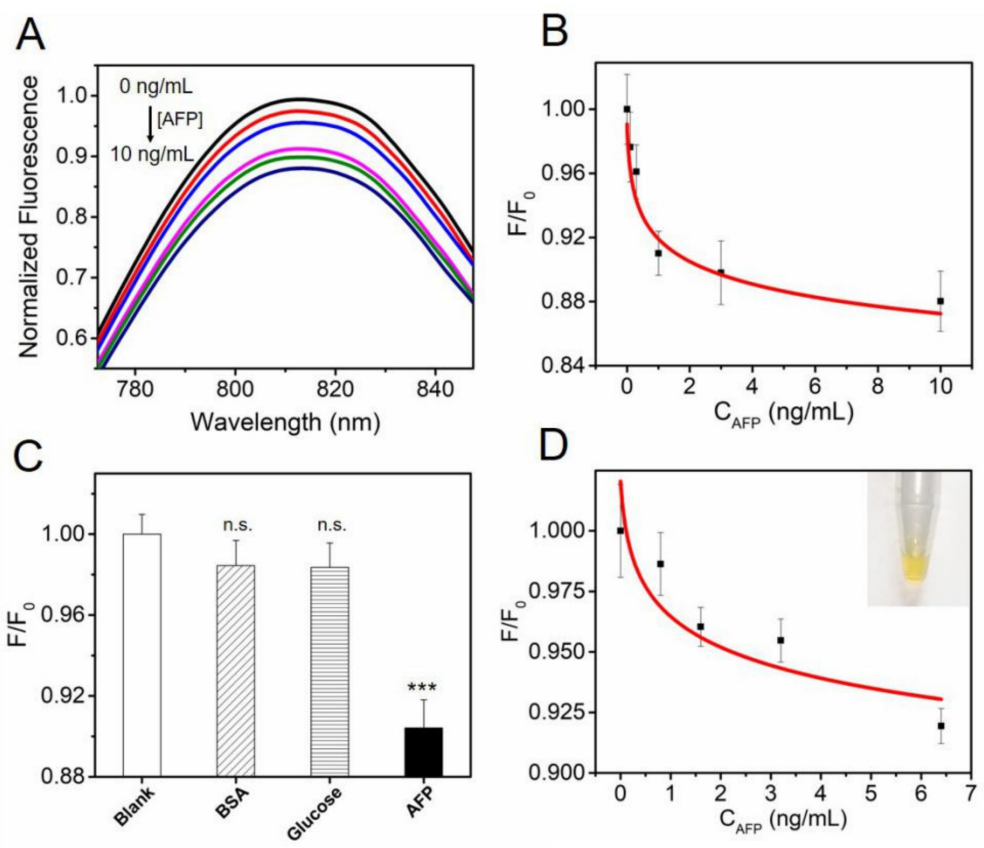

Figure 4. Sensitivity and selectivity of as-prepared Au NR-QDs apta-sensor for AFP detection. (A) Normalized fluorescence spectra of Au NR-QDs apta-sensor incubated with different concentrations of AFP from 0 to $10 \mathrm{ng} / \mathrm{mL}$, where the maximum fluorescence intensity of Au NR-QDs apta-sensor was regarded as 1.0. (B) The relative fluorescence intensities $\left(F / F_{0}\right)$ against the AFP concentration exacted from (A). (C) The variation of $F / F_{0}$ for Au NR-QDs apta-sensor treated with PBS (blank), bull serum albumin (BSA, $1 \mu \mathrm{g} / \mathrm{mL})$, glucose $(100 \mathrm{ng} / \mathrm{mL})$, and AFP $(10 \mathrm{ng} / \mathrm{mL})$, respectively. The asterisks indicated the significant differences between blank and experiment groups ( ${ }^{* * *} p<0.001$, n.s. $p>0.05$ ). (D) Detection of AFP in spiked human serum with as-prepared Au NR-QDs apta-sensor. The relative fluorescence intensities $\left(F / F_{0}\right)$ showed a similar negative relationship against AFP concentration in human serums. The inset is the picture of human serum.

Table 1. The application of gold nanorods-quantum dots (Au NR-QDs) apta-sensor for the determination of serum samples spiked with different concentrations of alpha fetoprotein (AFP).

\begin{tabular}{ccccc}
\hline Sample & $\begin{array}{c}\text { Concentration of AFP } \\
\text { Spiked }(\mathbf{n g} / \mathbf{m L})\end{array}$ & $\begin{array}{c}\text { Concentration of AFP } \\
\text { Found }(\mathbf{n g} / \mathbf{m L})\end{array}$ & $\begin{array}{c}\text { Recovery } \\
\mathbf{( \% )}\end{array}$ & $\begin{array}{c}\text { RSD } \\
\mathbf{( \% )}\end{array}$ \\
\hline diluted serum 1 & 1.6 & 1.9 & 118 & 9.48 \\
diluted serum 2 & 6.4 & 7.3 & 114 & 7.18 \\
diluted serum 3 & 10 & 10.40 & 104 & 2.33 \\
\hline
\end{tabular}

\subsection{Construction of $A u N R-Q D$ s and $A u N P-Q D$ s Nano-sensors for cTnI Detection}

To demonstrate the universality of the plasmon-regulated FRET sensing strategy with random modification layers and plasmonic nanostructures, antibodies modified nano-sensors for cardiac troponin I (cTnI) detection were constructed based on Au NR-QDs and Au nano-prism-QDs 
(Au NP-QDs), respectively. An Au NR-QDs-Ab nano-sensor against cTnI was constructed as shown in Figure S11. A similar sensing performance was observed $\left(R^{2}=0.99\right)$ for the Au NR-QDs-Ab nano-sensor with a LOD of $0.75 \mathrm{ng} / \mathrm{mL}$. Furthermore, with Au nano-prisms as plasmonic nanostructures, an Au NP-QDs-Ab nano-sensor was constructed as shown in Figure 5A. Antibodies can be also modified onto the surfaces of $\mathrm{Au}$ NPs by the crosslinking of $-\mathrm{NH}_{2}$. The absorption and fluorescence spectra for the fabrication process of Au NP-QDs-Ab nano-sensor is shown in Figure 5B,C. The linkage of QDs on the surfaces of Au NPs caused a $\sim 34 \mathrm{~nm}$ redshift of $\lambda_{\text {LSPR }}$ and a $\sim 60 \%$ decrease of fluorescence intensity. After the modification of antibodies on the surfaces of Au NP-QDs, another $\sim 11 \mathrm{~nm}$ redshift of $\lambda_{\mathrm{LSPR}}$ and $\sim 20 \%$ decrease of fluorescence intensity were observed. Then, the sensitivity of as-prepared Au NP-QDs nano-sensor for cTnI detection was demonstrated. A remarkable decrease of fluorescence intensity occurred with the increasing concentration of cTnI (Figure 5D). There was also a power function type relationship between $F / F_{0}$ and $c T n I$ concentration as we expected $\left(F / F_{0}=131.3\left(C_{c T n I}+17.00\right)^{-1.732}\right.$, $\left.R^{2}=0.95\right)$ with the LOD of $0.33 \mathrm{ng} / \mathrm{mL}$, which was lower than the critical concentration of cTnI $(2.0 \mathrm{ng} / \mathrm{mL})$ in healthy human serum (Figure 5E) [60]. The results suggest that the universality of our plasmon-FERT sensing system enables the detection for different analytes by changing the modification layer and/or plasmonic nanostructures.

A
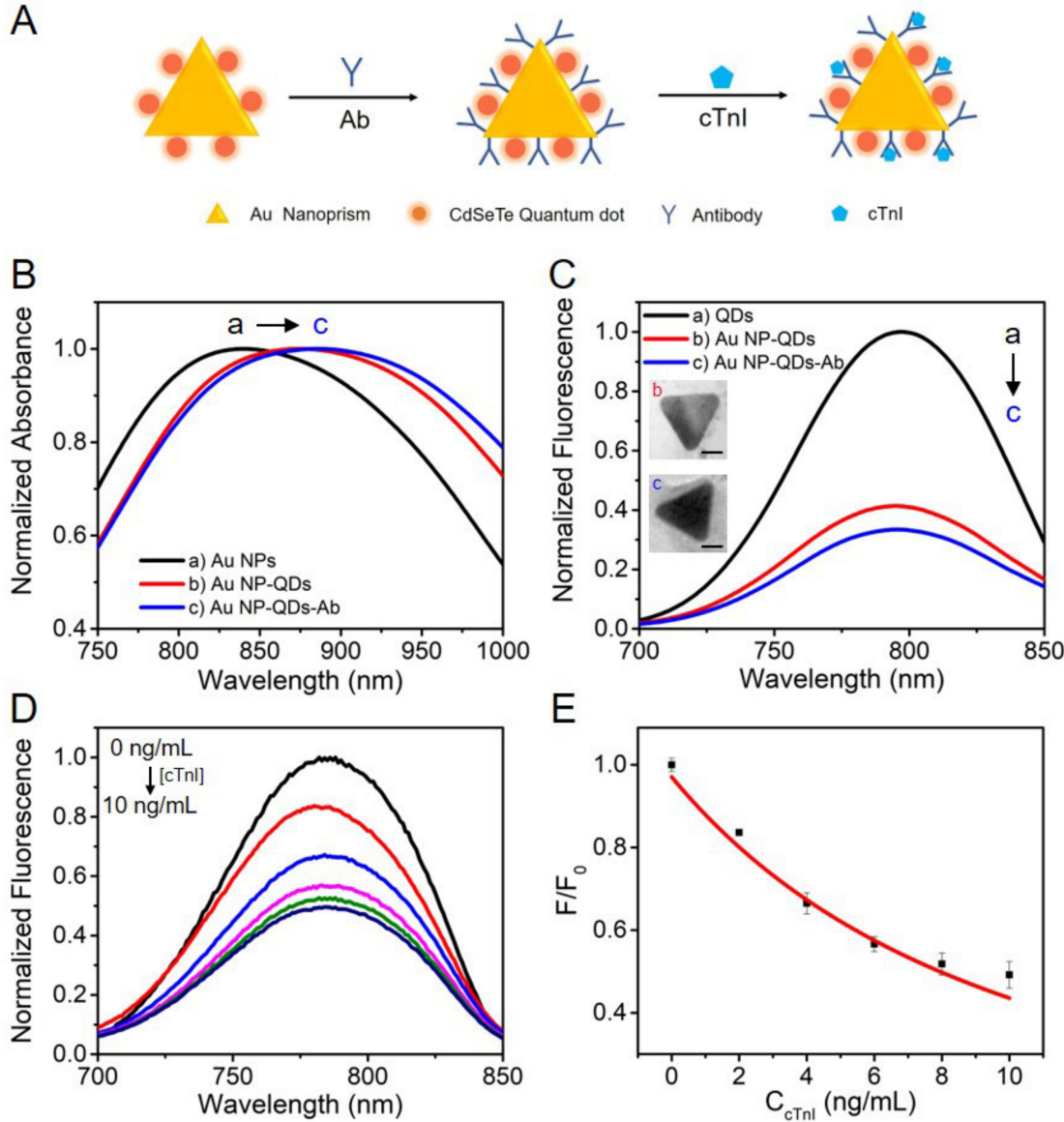

Figure 5. The antibodies-modified Au nano-prism-QDs (Au NP-QDs-Ab) nano-sensor for the detection of cardiac troponin I (cTnI). (A) Schematics for the construction of the Au NP-QDs-Ab nano-sensor and its application for CTnI detection. (B) Normalized UV-vis absorption spectra and (C) normalized fluorescence spectra during the fabrication process of the Au NP-QDs nano-sensor. The insets in (C) were the TEM images of Au NP-QDs (b) and Au NP-QDs-Ab (c). Scale bars are $20 \mathrm{~nm}$. (D) Normalized fluorescence spectra of Au NP-QDs-Ab nano-sensor incubated with different concentrations of cTnI ranging from 0 to $10 \mathrm{ng} / \mathrm{mL}$, where the maximum fluorescence intensity of Au NP-QDs sensor was regarded as 1.0. (E) The relationship of relative fluorescence intensities $\left(F / F_{0}\right)$ against cTnI concentration $\left(F / F_{0}=131.3\left(C_{c T n I}+17.00\right)^{-1.732}, R^{2}=0.95\right)$ extracted from $(D)$. 


\section{Conclusions}

In summary, we have designed a plasmon-regulated FRET sensing system of Au NR-QDs with regulated $E_{t}$ for sensitive and selective detection of biomarkers. The regulated $E_{t}$ arises from the tunable LSPR properties of plasmonic nanostructures against the RI change. It was first reported that the absorption spectra of the plasmonic structure in a plasmon-emitter hybrid nanostructure were employed to regulate $E_{t}$ and further applied for analytes detection. This plasmon-regulated FRET sensing system possesses the following unique features: (1) good sensing reversibility, (2) effectively improved sensitivity, compared with the sensing performance of the plasmonic substrate based on the shift of LSPR, (3) the ability for serving as a universal sensing platform for the detection of various analytes due to the simple and flexible fabrication and modification process. Certainly, the present work was still confronted with some limitations. For example, the sensitivity for the detection of analytes in a wide concentration range is limited, and the anti-interference ability needs to be improved. Further studies will aim to increase the selectivity and try to realize the high throughput sensing of multiplex analytes by simultaneously introducing various fluorescent materials with different emission peaks into one plasmon-regulated FRET nano-sensor. In all, our proposed plasmon-regulated FRET strategy is encouraging to develop general sensing platforms with the advantages of easy fabrication and high sensitivity for high-throughput, real-time, label-free, and multi-analyte sensing applications in the field of chemical and biological analysis.

Supplementary Materials: The following are available online at http://www.mdpi.com/2079-4991/10/3/444/s1. Figure S1: UV-vis spectra of Au NRs before and after the modification of cysteamine. Figure S2: Fabrication of Au NR-QDs assemblies using Au NRs with different LSPR peak wavelength. Figure S3: Fabrication of Au NR-QDs assemblies using QDs of different concentrations. Figure S4: The monitoring for the fabrication process of Au NR-QDs assemblies. Figure S5: TEM images of QDs and Au NRs. Table S1: Zeta-potentials of different structures during the fabrication of Au NR-QDs. Figure S6: The response of Au NRs to RI change. Figure S7: The fluorescence stability of QDs against RI. Figure S8: Sensing reversibility and stability of Au NR-QDs upon reversible changes of RI. Figure S9: The fluorescence stability of the Au NR-QDs apta-sensor. Figure S10: Au NRs apta-sensor for AFP detection from 0.1 to $50 \mathrm{ng} / \mathrm{mL}$. Figure S11: Construction of an antibodies-modified Au NR-QDs sensor for cTnI detection.

Author Contributions: Conceptualization, J.Z. Investigation and methodology, X.L., Y.W., Q.F., and Y.W. Writing - original draft preparation, X.L. and Y.W. Writing - review and editing, J.Z., D.M., and B.Z. Funding acquisition, J.Z. All authors have read and agreed to the published version of the manuscript.

Funding: The National Natural Science Foundation of China (No. 21775168), National Key R\&D Program of China (No. 2017YFE0102400), Department of Science and Technology of Guangdong Province (No. 2017A020211004), Shenzhen Research Funding Program (No. JCYJ20180307155036127), and Fundamental Research Funds for the Central Universities (No. 191gpy211) partly supported this work. The China Scholarship Council also partly supported this work.

Conflicts of Interest: The authors declare no conflict of interest.

\section{References}

1. Maccaferri, N.; Zhao, Y.Q.; Isoniemi, T.; Iarossi, M.; Parracino, A.; Strangi, G.; De Angelis, F. Hyperbolic meta-antennas enable full control of scattering and absorption of light. Nano Lett. 2019, 19, 1851-1859. [CrossRef] [PubMed]

2. Shen, Y.; Zhou, J.H.; Liu, T.R.; Tao, Y.T.; Jiang, R.B.; Liu, M.X.; Xiao, G.H.; Zhu, J.H.; Zhou, Z.K.; Wang, X.H.; et al. Plasmonic gold mushroom arrays with refractive index sensing figures of merit approaching the theoretical limit. Nat. Commun. 2013, 4, 2381. [CrossRef] [PubMed]

3. Mayer, K.M.; Hafner, J.H. Localized surface plasmon resonance sensors. Chem. Rev. 2011, 111, 3828-3857. [CrossRef] [PubMed]

4. Tang, L.H.; Li, J.H. Plasmon-based colorimetric nanosensors for ultrasensitive molecular diagnostics. ACS Sens. 2017, 2, 857-875. [CrossRef] [PubMed]

5. Anker, J.N.; Hall, W.P.; Lyandres, O.; Shah, N.C.; Zhao, J.; Van Duyne, R.P. Biosensing with plasmonic nanosensors. Nat. Mater. 2008, 7, 442-453. [CrossRef] 
6. Sreekanth, K.V.; Alapan, Y.; ElKabbash, M.; Ilker, E.; Hinczewski, M.; Gurkan, U.A.; De Luca, A.; Strangi, G. Extreme sensitivity biosensing platform based on hyperbolic metamaterials. Nat. Mater. 2016, 15, 621-628. [CrossRef]

7. Liu, B.W.; Chen, S.; Zhang, J.C.; Yao, X.; Zhong, J.H.; Lin, H.X.; Huang, T.X.; Yang, Z.L.; Zhu, J.F.; Liu, S.; et al. A plasmonic sensor array with ultrahigh figures of merit and resonance linewidths down to $3 \mathrm{~nm}$. Adv. Mater. 2018, 30, 1706031. [CrossRef]

8. Wang, Y.Y.; Zhou, J.H.; Li, J.H. Construction of plasmonic nano-biosensor-based devices for point-of-care testing. Small Methods 2017, 1, 1700197. [CrossRef]

9. Szunerits, S.; Boukherroub, R. Sensing using localised surface plasmon resonance sensors. Chem. Commun. 2012, 48, 8999-9010. [CrossRef]

10. Culver, H.R.; Wechsler, M.E.; Peppas, N.A. Label-free detection of tear biomarkers using hydrogel-coated gold nanoshells in a localized surface plasmon resonance-based biosensor. ACS Nano 2018, 12, 9342-9354. [CrossRef]

11. Li, W.; Jiang, X.; Xue, J.; Zhou, Z.; Zhou, J. Antibody modified gold nano-mushroom arrays for rapid detection of alpha-fetoprotein. Biosens. Bioelectron. 2015, 68, 468-474. [CrossRef]

12. Sabela, M.; Balme, S.; Bechelany, M.; Janot, J.M.; Bisetty, K. A review of gold and silver nanoparticle-based colorimetric sensing assays. Adv. Eng. Mater. 2017, 19, 1700270. [CrossRef]

13. Coglitore, D.; Janot, J.M.; Balme, S. Protein at liquid solid interfaces: Toward a new paradigm to change the approach to design hybrid protein/solid-state materials. Adv. Colloid Interface Sci. 2019, 270, 278-292. [CrossRef]

14. Li, J.F.; Li, C.Y.; Aroca, R.F. Plasmon-enhanced fluorescence spectroscopy. Chem. Soc. Rev. 2017, 46, 3962-3979. [CrossRef]

15. Kim, J.E.; Choi, J.H.; Colas, M.; Kim, D.H.; Lee, H. Gold-based hybrid nanomaterials for biosensing and molecular diagnostic applications. Biosens. Bioelectron. 2016, 80, 543-559. [CrossRef]

16. Wang, M.S.; Krasnok, A.; Zhang, T.Y.; Scarabelli, L.; Liu, H.; Wu, Z.L.; Liz-Marzan, L.M.; Terrones, M.; Alu, A.; Zheng, Y.B. Tunable Fano resonance and plasmon-exciton coupling in single Au nanotriangles on monolayer $\mathrm{WS}_{2}$ at room temperature. Adv. Mater. 2018, 30, 1705779. [CrossRef]

17. Luk'yanchuk, B.; Zheludev, N.I.; Maier, S.A.; Halas, N.J.; Nordlander, P.; Giessen, H.; Chong, C.T. The Fano resonance in plasmonic nanostructures and metamaterials. Nat. Mater. 2010, 9, 707-715. [CrossRef]

18. Ramirez, M.O.; Molina, P.; Gomez-Tornero, A.; Hernandez-Pinilla, D.; Sanchez-Garcia, L.; Carretero-Palacios, S.; Bausa, L.E. Hybrid plasmonic-ferroelectric architectures for lasing and SHG processes at the nanoscale. Adv. Mater. 2019, 31, 1901428. [CrossRef]

19. Rakovich, A.; Albella, P.; Maier, S.A. Plasmonic control of radiative properties of semiconductor quantum dots coupled to plasmonic ring cavities. ACS Nano 2015, 9, 2648-2658. [CrossRef]

20. Werschler, F.; Lindner, B.; Hinz, C.; Conradt, F.; Gumbsheimer, P.; Behovits, Y.; Negele, C.; de Roo, T.; Tzang, O.; Mecking, S.; et al. Efficient emission enhancement of single CdSe/CdS/PMMA quantum dots through controlled near-field coupling to plasmonic bullseye resonators. Nano Lett. 2018, 18, 5396-5400. [CrossRef]

21. Zhang, X.; Hu, Y.; Yang, X.; Tang, Y.; Han, S.; Kang, A.; Deng, H.; Chi, Y.; Zhu, D.; Lu, Y. Forster resonance energy transfer (FRET)-based biosensors for biological applications. Biosens. Bioelectron. 2019, 138, 111314. [CrossRef]

22. Ling, J.A.; Huang, C.Z. Energy transfer with gold nanoparticles for analytical applications in the fields of biochemical and pharmaceutical sciences. Anal. Methods 2010, 2, 1439-1447. [CrossRef]

23. Bitton, O.; Gupta, S.N.; Haran, G. Quantum dot plasmonics: From weak to strong coupling. Nanophotonics 2019, 8, 559-575. [CrossRef]

24. Lee, J.H.; Cho, H.Y.; Choi, H.K.; Lee, J.Y.; Choi, J.W. Application of gold nanoparticle to plasmonic biosensors. Int. J. Mol. Sci. 2018, 19, 2021. [CrossRef]

25. Jeong, Y.; Kook, Y.M.; Lee, K.; Koh, W.G. Metal enhanced fluorescence (MEF) for biosensors: General approaches and a review of recent developments. Biosens. Bioelectron. 2018, 111, 102-116. [CrossRef]

26. Abadeer, N.S.; Brennan, M.R.; Wilson, W.L.; Murphy, C.J. Distance and plasmon wavelength dependent fluorescence of molecules bound to silica-coated gold nanorods. ACS Nano 2014, 8, 8392-8406. [CrossRef]

27. Aissaoui, N.; Moth Poulsen, K.; Kall, M.; Johansson, P.; Wilhelmsson, L.M.; Albinsson, B. FRET enhancement close to gold nanoparticles positioned in DNA origami constructs. Nanoscale 2017, 9, 673-683. [CrossRef] 
28. Fang, A.J.; Chen, H.Y.; Li, H.T.; Liu, M.L.; Zhang, Y.Y.; Yao, S.Z. Glutathione regulation-based dual-functional upconversion sensing-platform for acetylcholinesterase activity and cadmium ions. Biosens. Bioelectron. 2017, 87, 545-551. [CrossRef]

29. Xia, Y.; Song, L.; Zhu, C. Turn-on and near-infrared fluorescent sensing for 2,4,6-trinitrotoluene based on hybrid (gold nanorod)-(quantum dots) assembly. Anal. Chem. 2011, 83, 1401-1407. [CrossRef]

30. Qu, A.H.; Xu, L.G.; Sun, M.Z.; Liu, L.Q.; Kuang, H.; Xu, C.L. Photoactive hybrid AuNR-Pt@Ag 2 S core-satellite nanostructures for near-infrared quantitive cell imaging. Adv. Funct. Mater. 2017, 27, 1703408. [CrossRef]

31. He, W.; Sun, X.; Liu, B.; Shen, J. A label-free "SEF-FRET" fluorescent sensing platform for ultrasensitive DNA detection based on AgNPs SAMs. Talanta 2019, 205, 120072. [CrossRef]

32. Zhou, L.; Ji, F.; Zhang, T.; Wang, F.; Li, Y.; Yu, Z.; Jin, X.; Ruan, B. An fluorescent aptasensor for sensitive detection of tumor marker based on the FRET of a sandwich structured QDs-AFP-AuNPs. Talanta 2019, 197, 444-450. [CrossRef]

33. Xu, D.D.; Liu, C.; Li, C.Y.; Song, C.Y.; Kang, Y.F.; Qi, C.B.; Lin, Y.; Pang, D.W.; Tang, H.W. Dual amplification fluorescence assay for alpha fetal protein utilizing immunohybridization chain reaction and metal-enhanced fluorescence of carbon nanodots. ACS Appl. Mater. Interfaces 2017, 9, 37606-37614. [CrossRef]

34. Kim, E.J.; Kim, E.B.; Lee, S.W.; Cheon, S.A.; Kim, H.J.; Lee, J.; Lee, M.K.; Ko, S.; Park, T.J. An easy and sensitive sandwich assay for detection of Mycobacterium tuberculosis Ag85B antigen using quantum dots and gold nanorods. Biosens. Bioelectron. 2017, 87, 150-156. [CrossRef]

35. Yang, S.H.; Zhang, F.F.; Wang, Z.H.; Liang, Q.L. A graphene oxide-based label-free electrochemical aptasensor for the detection of alpha-fetoprotein. Biosens. Bioelectron. 2018, 112, 186-192. [CrossRef]

36. Haran, G.; Chuntonov, L. Artificial plasmonic molecules and their interaction with real molecules. Chem. Rev. 2018, 118, 5539-5580. [CrossRef]

37. Li, M.; Cushing, S.K.; Wang, Q.Y.; Shi, X.D.; Hornak, L.A.; Hong, Z.L.; Wu, N.Q. Size-dependent energy transfer between CdSe/ZnS quantum dots and gold nanoparticles. J. Phys. Chem. Lett. 2011, 2, 2125-2129. [CrossRef]

38. Bujak, L.; Ishii, T.; Sharma, D.K.; Hirata, S.; Vacha, M. Selective turn-on and modulation of resonant energy transfer in single plasmonic hybrid nanostructures. Nanoscale 2017, 9, 1511-1519. [CrossRef]

39. Yu, M.Q.; Wang, H.; Fu, F.; Li, L.Y.; Li, J.; Li, G.; Song, Y.; Swihart, M.T.; Song, E.Q. Dual-recognition Forster resonance energy transfer based platform for one-step sensitive detection of pathogenic bacteria using fluorescent vancomycin-gold nanoclusters and aptamer-gold nanoparticles. Anal. Chem. 2017, 89, 4085-4090. [CrossRef]

40. Huang, C.J.; Lin, H.I.; Shiesh, S.C.; Lee, G.B. An integrated microfluidic system for rapid screening of alpha-fetoprotein-specific aptamers. Biosens. Bioelectron. 2012, 35, 50-55. [CrossRef]

41. Nikoobakht, B.; El Sayed, M.A. Preparation and growth mechanism of gold nanorods (NRs) using seed-mediated growth method. Chem. Mater. 2003, 15, 1957-1962. [CrossRef]

42. Wang, C.; Irudayaraj, J. Gold nanorod probes for the detection of multiple pathogens. Small 2008, 4, $2204-2208$. [CrossRef]

43. Maity, A.R.; Stepensky, D. Efficient subcellular targeting to the cell nucleus of quantum dots densely decorated with a nuclear localization sequence peptide. ACS Appl. Mater. Interfaces 2016, 8, 2001-2009. [CrossRef]

44. Shi, Y.; Zhang, H.; Yue, Z.; Zhang, Z.; Teng, K.S.; Li, M.J.; Yi, C.; Yang, M. Coupling gold nanoparticles to silica nanoparticles through disulfide bonds for glutathione detection. Nanotechnology 2013, 24, 375501. [CrossRef]

45. Wang, C.; Qian, J.; Wang, K.; Yang, X.; Liu, Q.; Hao, N.; Wang, C.; Dong, X.; Huang, X. Colorimetric aptasensing of ochratoxin A using $\mathrm{Au} @ \mathrm{Fe}_{3} \mathrm{O}_{4}$ nanoparticles as signal indicator and magnetic separator. Biosens. Bioelectron. 2016, 77, 1183-1191. [CrossRef]

46. Wu, H.Y.; Huang, W.L.; Huang, M.H. Direct high-yield synthesis of high aspect ratio gold nanorods. Cryst. Growth Des. 2007, 7, 831-835. [CrossRef]

47. Haldar, K.K.; Sen, T.; Patra, A. Metal conjugated semiconductor hybrid nanoparticle-based fluorescence resonance energy transfer. J. Phys. Chem. C 2010, 114, 4869-4874. [CrossRef]

48. Stanisavljevic, M.; Krizkova, S.; Vaculovicova, M.; Kizek, R.; Adam, V. Quantum dots-fluorescence resonance energy transfer-based nanosensors and their application. Biosens. Bioelectron. 2015, 74, 562-574. [CrossRef]

49. Gaur, G.; Koktysh, D.S.; Weiss, S.M. Immobilization of quantum dots in nanostructured porous silicon films: Characterizations and signal amplification for dual-mode optical biosensing. Adv. Funct. Mater. 2013, 23, 3604-3614. [CrossRef] 
50. Link, S.; Mohamed, M.B.; El-Sayed, M.A. Simulation of the optical absorption spectra of gold nanorods as a function of their aspect ratio and the effect of the medium dielectric constant. J. Phys. Chem. B 1999, 103, 3073-3077. [CrossRef]

51. Cui, M.; Wang, Y.; Jiao, M.; Jayachandran, S.; Wu, Y.; Fan, X.; Luo, X. Mixed self-assembled aptamer and newly designed zwitterionic peptide as antifouling biosensing interface for electrochemical detection of alpha-fetoprotein. ACS Sens. 2017, 2, 490-494. [CrossRef] [PubMed]

52. Breaker, R.R. Natural and engineered nucleic acids as tools to explore biology. Nature 2004, 432, 838-845. [CrossRef]

53. Song, S.P.; Wang, L.H.; Li, J.; Zhao, J.L.; Fan, C.H. Aptamer-based biosensors. Trends Anal. Chem. 2008, 27, 108-117. [CrossRef]

54. Wang, W.T.; Wang, W.; Davis, J.J.; Luo, X.L. Ultrasensitive and selective voltammetric aptasensor for dopamine based on a conducting polymer nanocomposite doped with graphene oxide. Microchim. Acta 2015, 182, 1123-1129. [CrossRef]

55. Coglitore, D.; Giamblanco, N.; Kizalaite, A.; Coulon, P.E.; Charlot, B.; Janot, J.M.; Balme, S. Unexpected hard protein behavior of BSA on gold nanoparticle caused by resveratrol. Langmuir 2018, 34, 8866-8874. [CrossRef] [PubMed]

56. Zhang, Y.L.; Qi, S.J.; Liu, Z.G.; Shi, Y.P.; Yue, W.Q.; Yi, C.Q. Rapid determination of dopamine in human plasma using a gold nanoparticle-based dual-mode sensing system. Mater. Sci. Eng. C 2016, 61, 207-213. [CrossRef] [PubMed]

57. Hu, W.W.; Chen, Q.S.; Li, H.H.; Ouyang, Q.; Zhao, J.W. Fabricating a novel label-free aptasensor for acetamiprid by fluorescence resonance energy transfer between $\mathrm{NH}_{2}-\mathrm{NaYF}_{4}: \mathrm{Yb}, \mathrm{Ho}_{\mathrm{SiO}}$ and $\mathrm{Au}$ nanoparticles. Biosens. Bioelectron. 2016, 80, 398-404. [CrossRef]

58. Mock, J.J.; Hill, R.T.; Tsai, Y.J.; Chilkoti, A.; Smith, D.R. Probing dynamically tunable localized surface plasmon resonances of film-coupled nanoparticles by evanescent wave excitation. Nano Lett. 2012, 12, 1757-1764. [CrossRef]

59. Liu, Z.; Lee, H.; Xiong, Y.; Sun, C.; Zhang, X. Far-field optical hyperlens magnifying sub-diffraction-limited objects. Science 2007, 315, 1686. [CrossRef]

60. Han, X.; Li, S.H.; Peng, Z.L.; Othman, A.M.; Leblanc, R. Recent development of cardiac troponin I detection. ACS Sens. 2016, 1, 106-114. [CrossRef] 